\title{
Administration via Jejunostomy
}

National Cancer Institute

\section{Source}

National Cancer Institute. Administration via Jejunostomy. NCI Thesaurus. Code C38202.

Administration of a drug through a surgical opening between the jejunum and the surface of the abdominal wall. 